\title{
SUBNANOSECOND LASER FLUOROMETER WITH COMPACT NITROGEN LASER PUMPED DYE LASER FOR LIFETIME MEASUREMENTS
}

\author{
Kouichi ISHIBASHI, Tsukasa SHIGEZUMI, Totaro IMASAKA \\ and Nobuhiko ISHIBASHI ${ }^{\circledR}$ \\ Faculty of Engineering, Kyushu University \\ Hakozaki, Higashi-ku, Fukuoka 812
}

\begin{abstract}
A small atmospheric pressure nitrogen laser was constructed. This laser produced $20 \mu \mathrm{J}$ pulses at $337.1 \mathrm{~nm}$ and had performances of pulse widths of $660 \mathrm{ps}$ and a maximum repetition rate of $80 \mathrm{~Hz}$. Lifetime measurements of nanosecond fluorescence decay were demonstrated by using the fluorometric system consisting of a nitrogen laser pumped dye 1 aser ( $<250$ ps FWHM) and a fast microchannel plate photomultiplier. The time resolution of the system was $630 \mathrm{ps}$. The lifetimes of acridine, perylene, benzo(k)fluoranthene, and benzo(a)pyrene were determined to be $1.0,6,12$, and $35 \mathrm{~ns}$ without any deconvolution procedure. The lifetime measurement could be carried out at a concentration of $3 \times 10^{-10} \mathrm{M}$.
\end{abstract}

\section{INTRODUCTION}

A time-resolved fluorometer is one of the most important analytical tool for studying physical and chemical properties of fluorescent organic molecules. In this technique, a light source with short pulse widths is essential for the measurement of fluorescence decay. Furthermore, time-resolved fluorometry is useful for temporal discrimination of fluorescent samples from the large background noise occurring from Raman scattering and impurity fluorescence ${ }^{1)}$. A flashlamp, which is currently used in a conventional time-resolved fluorometer, has pulse widths of $2 \sim 6 \mathrm{~ns}$. By optimizing on operating voltage, the generation of subnanosecond pulses(800 ps) has been achieved. However, the pulse energy decreases rapidly with decreasing the pulse widths of the flashlamp. A laser has distinct advantages over the flashlamp with respect to short pulse widths and high photon flux. Nitrogen laser pumped dye lasers have found wide-spread applications in time-resolved fluorometry. The reduced pressure nitrogen laser and its pumped dye lasers have a large pulse energy, but they have usually pulse widths of several nanoseconds( $3 \sim 10 \mathrm{~ns})$. Unfortunately, it is not sufficiently short for the lifetime measurement of the organic molecules in the condensed phase(1 10 ns). The generation of subnanosecond dye laser pulses has been achieved based on the idea to use a transversely excited atmospheric(TEA) pressure nitrogen laser as a pumping source ${ }^{2}$ ) 6 ). This type of the nitrogen laser is 
recently commercially available, but it is still expensive and complicated. This paper describes the construction of a very compact TEA nitrogen laser and its performances and presents a few applications to the lifetime measurements using the fluorometer consisting of a subnanosecond dye laser source pumped by a TEA nitrogen laser and a fast microchannel plate photomultiplier.

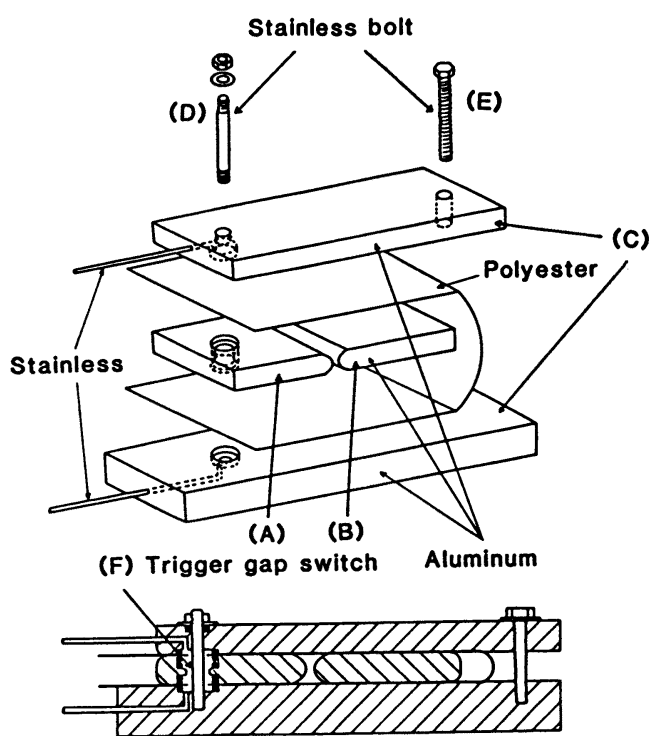

Fig. 1 Mechanical construction of nitrogen laser

\section{EXPERIMENTAL}

TEA nitrogen laser

The TEA nitrogen laser constructed in this study is shown in Fig. 1. The basic element is a parallel plate transmission line of the Blumlein type. The discharge electrodes (A,B) made of aluminum are $50 \mathrm{~mm}$ long, $50 \mathrm{~mm}$ wide, and $10 \mathrm{~mm}$ thick. These electrodes are tightly fastened with two stainless bolts (D,E) between two ground electrodes (C) insulated by a $0.25 \mathrm{~mm}$ thick polyester film. One of the bolts(D) acts as a ground electrode of a trigger gap switch(F). The position of the electrode(B) is adjusted by screws from the outside to optimize the distance between the discharge electrodes ( $1 \sim 4 \mathrm{~mm}$ ). The characteristic impedance and the capacitance of the transmission line are $0.52 \Omega$ and $0.53 \mathrm{nF}$, respectively. The nitrogen laser is shielded against a

large quantity of the radiofrequency interference by an aluminum box ( $140 \mathrm{~mm} \times 200$ $\mathrm{mm} \times 60 \mathrm{~mm}$ ), which has two quartz glass windows in front and back of the discharge channel. The back window is equipped with a mirror to feed back spontaneous emission. The present nitrogen laser is very simple and compact, and may be easily constructed in the usual laboratory with no technical supports.

Power supply

The nitrogen laser requires a power supply which generates a high voltage up to $20 \mathrm{kV}$. In order to reduce electrical shock hazards the laser line should be charged only for a short time, therefore a high voltage pulser is constructed and used in this study. The block diagrams of an electric circuits for a pulse generator(A) and a high voltage pulser(B) are shown in Fig. 2. The repetitive pulses generated by an unijunction transister(a) drives a small thyrister(b). The repetition rate is controlled by the time constant of the resister(c) and the capacitor(d). This electric pulse is fed to a gate part of a large thyrister(e). The primary capacitor(f) is charged by a direct current(DC) power supply, and its stored energy is instant1y discharged by the thyrister(e) and transfered to the 


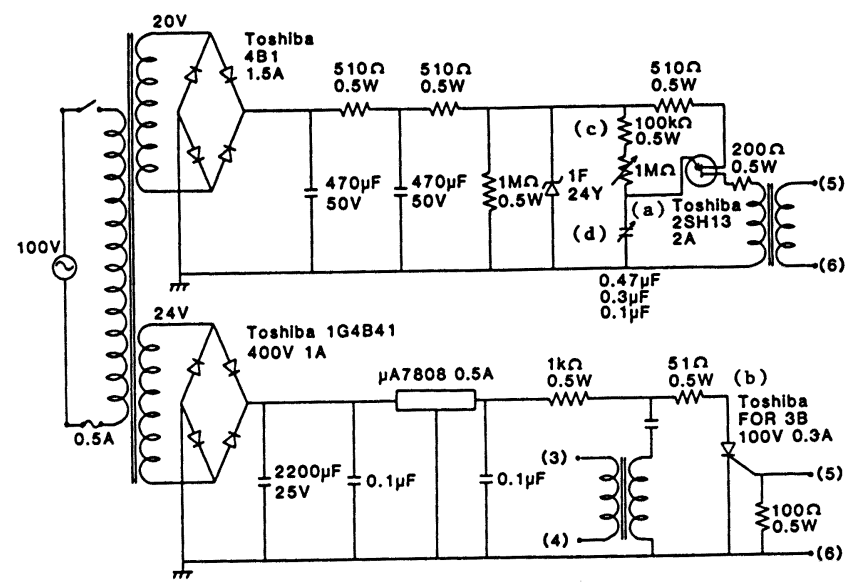

(A) Pulse generator

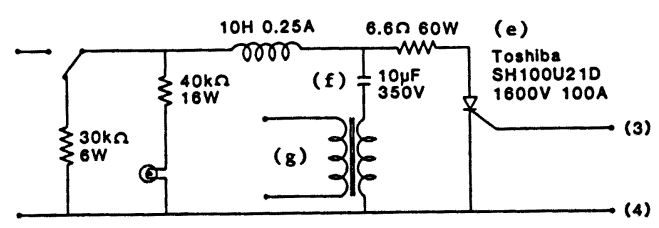

(B) High-voltage pulser

Fig. 2 Electric circuit for a pulse generator(A) and a high-voltage pulser(B) capacitor of the nitrogen laser by an ignition coil $(\mathrm{g})$ for a car engine (Nippondenso GT Hideluxe Coil). The break down voltage is determined by the voltage of the DC power supply and the pressure of the nitrogen gas in the trigger gap switch. It is noted that a very simple high voltage pulser may be available when the repetition rate is specified to $50 \mathrm{~Hz}^{7}$ ).

\section{Lifetime measurement}

A schematic diagram of a time-resolved fluorometer constructed in this study is shown in Fig. 3. The nitrogen laser pumps a laser dye of 4,4'-bis (buthyloctyloxy)p-quaterpheny $1(B B Q)$ and generates $386 \mathrm{~nm}$ laser emissison. The dye laser beam is split by a quartz plate, and the reflected laser pulse is detected by a biplanar

phototube (HTV, R1328U-02) to provide a trigger pulse for a sampling oscilloscope (Iwatsu, SS601B, $6 \mathrm{GHz}$ ). The transmitted laser beam is focused by a lens(Asahi, focal length $40 \mathrm{~cm}$ ) into a sample cell from a bottom window. The sample cell is a conventional quartz cuvette with transparent quartz windows. Fluorescence from a sample solution is focused by a glass lens(focal length $35 \mathrm{~cm}$, diameter

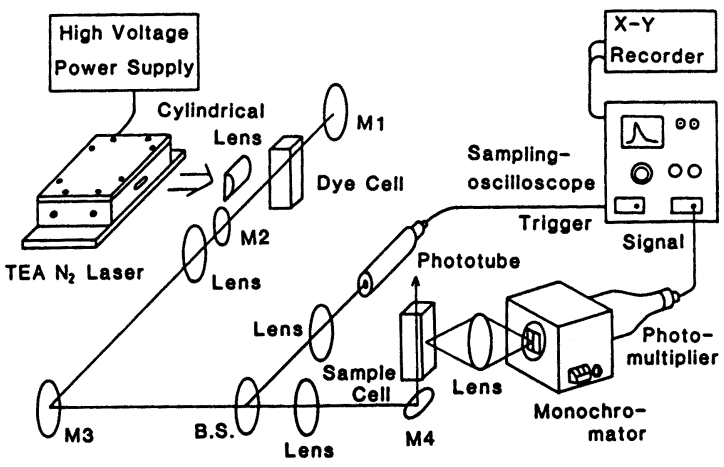

Fig. 3 Schematic diagram of laser fluorometer
$40 \mathrm{~mm}$ ) onto the entrance slit of a monochromator (Jasco, CT-10, dispersion $8 \mathrm{~nm} \mathrm{~mm}{ }^{-1}$, $500 \mathrm{~nm}$ blaze) equipped with a microchannel plate photomutiplier(HTV, R1294U-01, $1 \mathrm{GHz}$ ). The signal is introduced into the SH-3B head of the sampling oscilloscope. The output signal from the oscilloscope is plotted by a $X-Y$ recorder (National, VP-6421A). 
Reagents and procedure

Benzo(a)pyrene, benzo(k)fluoranthene, and perylene were obtained from Wako Pure Chemicals and acridine from Kishida Chemical Co. These reagents were used without further purification. Benzene and methanol used as solvents were of fluorescence spectroscopy grade obtained from E. Merk AG. Stock solutions $\left(10^{-3} \mathrm{M}\right)$ were prepared by dissolving the reagents into benzene at first, and were diluted stepwise with methanol down to the specified concentrations $\left(10^{-4} \sim 10^{-10} \mathrm{M}\right)$.

\section{RESULTS AND DISCUSSION}

\section{TEA nitrogen laser}

The temporal profiles of the nitrogen and dye lasers were measured using the detection system consisting of a phototube and a sampling oscilloscope. The pulse widths of the nitrogen laser and the dye lasers were 660 ps and 250 ps, respectively. Since the time-resolution of the detection electronics is estimated to be about $250 \mathrm{ps}$, the pulse widths of the dye laser may be less than 250 ps. The obtained pulse shapes are lack of a long tail as observed in gaseous discharge light sources, so that they will be suitable light sources for time-resolved spectroscopic measurements. The separation of the discharge electrodes affected the pulse energy of the nitrogen laser, and stable operation was usually achieved at a separation of $2 \mathrm{~mm}$. The threshold level of 1 aser oscillation was $13 \mathrm{kV}$, and the pulse energy increased with the increasing applied voltage up to $22 \mathrm{kV}$ in this condition. Above this voltage the electronic energy stored in the capacitors leaked out through discharge to the ground electrodes and the shield box, and the laser action could not be obtained. A pulse energy of $10 \mu \mathrm{J}$ was obtained at $22 \mathrm{kV}$ and stability being 5 percent. When the separation of the discharge electrodes was adjusted to $4 \mathrm{~mm}$, the pulse energy increased up to $20 \mu \mathrm{J}$ in this condition and stability decreased down to 10 percent. The output energy per pulse and the average power were a function of the repetition rate of the laser. With increasing the repetition rate, the output energy decreased and the average power increased. A maximum repetition rate of $80 \mathrm{~Hz}$ under an average power

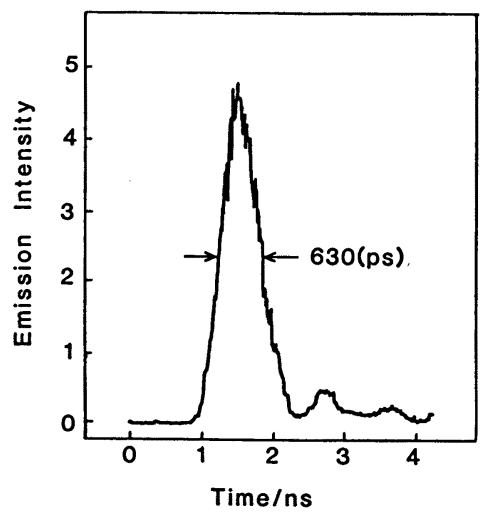

Fig. 4 Time resolution of laser fluorometric system of $400 \mu \mathrm{W}$ were achieved at an applied voltage of $21 \mathrm{kV}$ and an electrode separation of $2 \mathrm{~mm}$.

Lifetime measurements

A white paper was placed at the sample position, and the temporal profile of scattered laser emission was measured to observe the signal response of the present fluorometer. The result is shown in Fig. 4. The time resolution of the system is observed to be $630 \mathrm{ps}$. The weak undulation after the laser pulse may be a ringing of the photomultiplier.

The typical fluorescence decay curve of acridine in methanol is shown in Fig. 5. The semi-log plot of the decay curve gives the fluorescence lifetime of $1.0 \mathrm{~ns}$. This value 


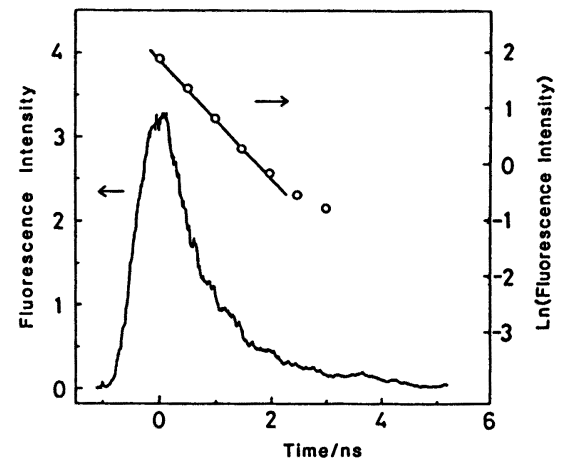

Fig. 5 F1uorescence decay curve of acridine $\left(7 \times 10^{-7} \mathrm{M}\right)$ $\lambda_{\mathrm{ex}}=386 \mathrm{~nm}, \lambda_{\mathrm{em}}=415 \mathrm{~nm}$
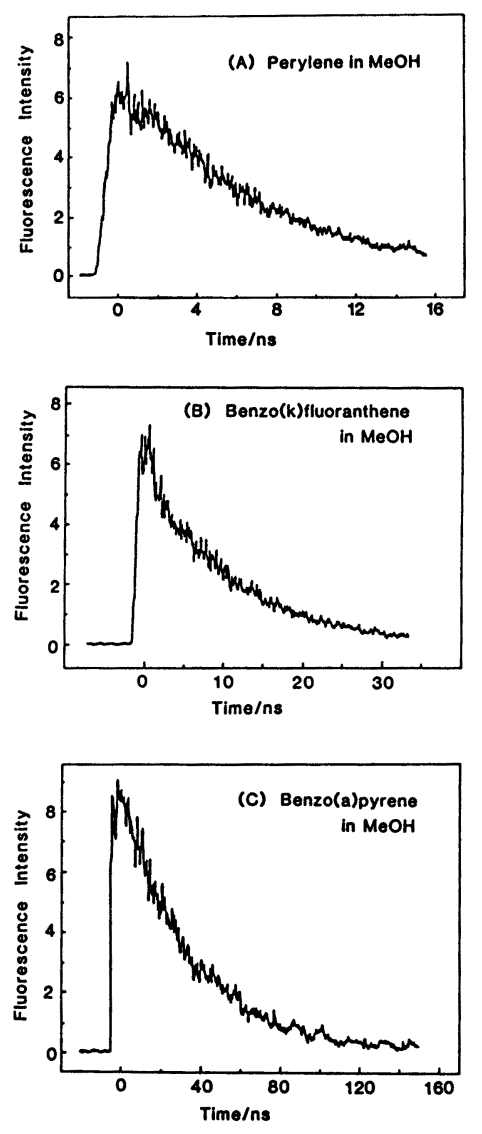

Fig. 6 Fluorescence decay curves $\lambda_{\mathrm{ex}}=386 \mathrm{~nm}$
(A) Perylene $\left(1 \times 10^{-7} \mathrm{M}\right), \lambda_{\text {em }}=437 \mathrm{~nm}$
(B) $\mathrm{B}(\mathrm{k}) \mathrm{F}\left(1 \times 10^{-7} \mathrm{M}\right), \lambda_{\mathrm{em}}=407 \mathrm{~nm}$
(C) B(a) Py $\left(1 \times 10^{-7} \mathrm{M}\right), \lambda_{\mathrm{em}}=405 \mathrm{~nm}$

almost agrees with the reference value of 0.9 ns for an ethanol solution in the Berlman's handbook ${ }^{8)}$. Love et al. have measured the fluorescence lifetime of acridine in ethanol using the time-correlated single photon counting system consisting of a flashlamp excitation source. They have obtained the fluorescence 1 ifetime of 1.7 ns from a graphical slope and the lifetime of $0.7 \mathrm{~ns}$ by deconvolution of the fluorescence decay curve ${ }^{9}$. Because of the poor time resolution of the fluorometer (FWHM $3.0 \mathrm{ns,} \mathrm{decay} \mathrm{time} 1.3 \mathrm{~ns}$ ), a deconvolution procedure is necessary for the flashlamp excitation method. On the other hand the present fluorometric system allows a direct determination of the florescence lifetime without any deconvolution. This point is a distinct advantage of the present laser excitation method. The noise in the decay curve originates mainly from instability of the output power of the nitrogen laser and partly from the radiofrequency interference by discharge.

The fluorescence decay curves of perylene, benzo(k)fluoranthene, and benzo(a)pyrene in methanol are shown in Fig. 6. The 1ifetimes of these three compounds were calculated by semi-log plots of the decay curves to be $6 \mathrm{~ns}$, $12 \mathrm{~ns}$, and $35 \mathrm{~ns}$, respectively. The value of

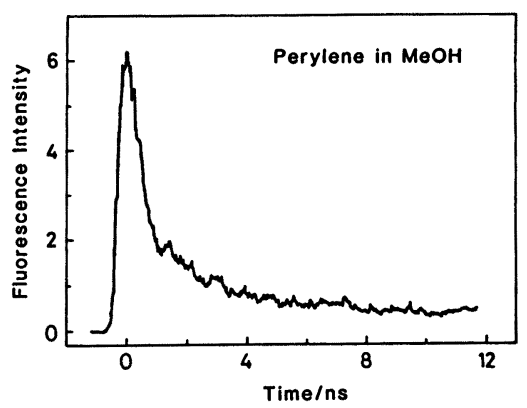

Fig. 7 Fluorescence decay curve of perylene $\left(3 \times 10^{-10} \mathrm{M}\right)$ $\lambda_{\mathrm{ex}}=386 \mathrm{~nm}, \lambda_{\mathrm{em}}=464 \mathrm{~nm}$ 
perylene agrees with the reference value of $0.4 \mathrm{~ns}$ for a cyclohexane solution fairly wel1 8 ).

Perylene has strong fluorescence bands at $437 \mathrm{~nm}$ and $464 \mathrm{~nm}$ when excited at $386 \mathrm{~nm}$. The fluorescence band at $437 \mathrm{~nm}$ is the largest one but a strong Raman band of methanol superimposes at this wavelength. The decay curve could not be clearly measured at $437 \mathrm{~nm}$ below $10^{-9} \mathrm{M}$ because of interference of Raman scattering. The fluorescence intensity at $464 \mathrm{~nm}$ is smaller than that at $437 \mathrm{~nm}$, but contribution of the Raman scattering is much smaller. So the decay curve of perylene can be readily measured at yet lower concentrations. Fig. 7 shows the decay curve of the $3 \times 10^{-10} \mathrm{M}$ solution of perylene. The peak at ou ns after excitation might be coming from Raman scattering and/or short-1ived impurity fluorescence, since a same profile was measured at 0 u $1 \mathrm{~ns}$ for the solvent only. The present dye laser has a relatively small output power, and it is disadvantageous as a light source in fluorometry. However, the fluorometric sensitivity is not determined by the output power of the dye laser but determined by the background signal from impurities in the solvent. The present laser may have a sufficient performance with respect to the output power in practical applications.

Comparison with other time-resolved fluorometer

The time-correlated photon counting technique with a flashlamp excitation source is currently used for fluorescence lifetime measurements. In this method the pulse width is rather large for the lifetime measurements and the pulse energy is not necessarily sufficient. So that the deconvolution procedure is necessary, and it requires a long time for recording a decay curve. This disadvantage makes it impossible to apply this technique to the detection system of high-performance liquid chromatography(HPLC).

A streak camera has a capability to measure the fluorescence decay curve instantly. It has a great temporal resolution(several picoseconds), and eliminates the need for deconvolution of the photomultiplier tube response. The exciting laser sources currently used are the mode-locked lasers. However, they are very expensive and may not be used in a conventional instrument. Walder and Winefordner have demonstrated the measurement of fluorescence decay curves of the sample at the several hundreds ppb levels with a fluorometric system consisting of a streak camera and a TEA nitrogen laser ${ }^{10)}$. However, this fluorometric system sometimes suffers from rather poor sensitivity for determination at ultratrace levels. Moreover, this system including a streak camera is considerably expensive.

There seems to be no alternative detection system for the conventional time-resolved fluorometer except for the combination of a fast photomultiplier and a sampling oscilloscope. It is emphasized that such a system can be used not only for lifetime measurements but also for the time-resolved signal detection in $\mathrm{HPLC}^{1)}$. Andreoni et al. have reported a time-resolved fluorometric system, which is similar to the present system, including a TEA nitrogen laser pumped dye laser and a sampling oscilloscope ${ }^{11)}$. They have not clarified the time resolution of the fluorometric system, but it may be in the order of $1 \mathrm{~ns}$ and similar to the present result. However, they have used a Varian 154M 
static cross field photomultiplier(400 ps, FWHM) for signal detection. Such a type of a photomultiplier is very expensive and its cost exceeds the price of a commercially available nitrogen laser and a sampling oscilloscope. Furthermore, present nitrogen laser is very simple and compact, and its physical dimension is $1 / 7$ of their instrument. Therefore, it might be useful as a conventiona1

fluorometer.

Many drugs are known to strongly fluoresce and have characteristic fluorescence lifetimes of $1 \sim 200 \mathrm{~ns}$. We expect that the fluorometric system described here may be quite useful for the lifetime measurements and trace analysis of these compounds.

\section{REFERENCES}

1) T. Imasaka, K. Ishibashi, N. Ishibashi: Ana1. Chim. Acta, 142, 1 (1982).

2) E. E. Bergman: Rev. Sci. Instrum., 48, 545 (1977).

3) E. E. Bergman: App1. Phys. Lett., 31, 661 (1977).

4) R. K. Bauer, A. Kowalczyk: Opt. Commun., 23, 169 (1977).

5) R. Cubeddu, S. De Silvestri: Opt. Quantum Electron., 11, 276 (1979).

6) T. Imasaka, N. Ishibashi: Ana1. Chem., 52, 2083 (1980) .

7) G. C. Bischoff, J. Matthews: Rev. Sci. Instrum., 49, 1602 (1978) .

8) I. B. Berlman: "Handbook of fluorescence spectra of aromatic molecules", p. 355, p. 399 (1971), (Academic Press, New York).

9) L. J. C. Love, L. A. Shaver: Ana1. Chem., 52, 154 (1980).

10) G. I. Walden, J. D. Winefordner: Spectrosc. Lett., 13, 785 (1980).

11) A. Andreoni, R. Cubeddu, S. De Silvestri, P. Laporta: Opt. Commun., $\underline{33}$, $277(1980)$.

Keyword phrases

atmospheric pressure nitrogen laser; time-resolved fluorometry; polycyclic aromatic hydrocarbons; fluorescence lifetime. 